Supplementary information - Retention of iodide and chloride by formation of a green rust solid solution GR-Cl $\mathrm{Cl}_{1-\mathrm{x}} \mathrm{I}_{\mathrm{x}}$ : A multi-scale approach

Tim Platte, ${ }^{* \mp}$ Nicolas Finck, ${ }^{\mp}$ Stefan Mangold, ${ }^{\square}$ Robert Polly, ${ }^{\mp}$ Horst Geckeis ${ }^{\mp}$

TInstitute for Nuclear Waste Disposal (INE), Karlsruhe Institute of Technology (KIT), P.O. Box 3640, 76021 Karlsruhe, Germany.

Institute for Photon Science and Synchrotron Radiation (IPS), Karlsruhe Institute of Technology (KIT), P.O. Box 3640, 76021 Karlsruhe, Germany. 


\section{X-ray diffraction}

Figure S1 shows the full diffractogram of all green rust (GR) samples. The diffractogram of all compounds indicate the presence of GR only, no presence of any other Fe-containing crystalline phase can be detected.

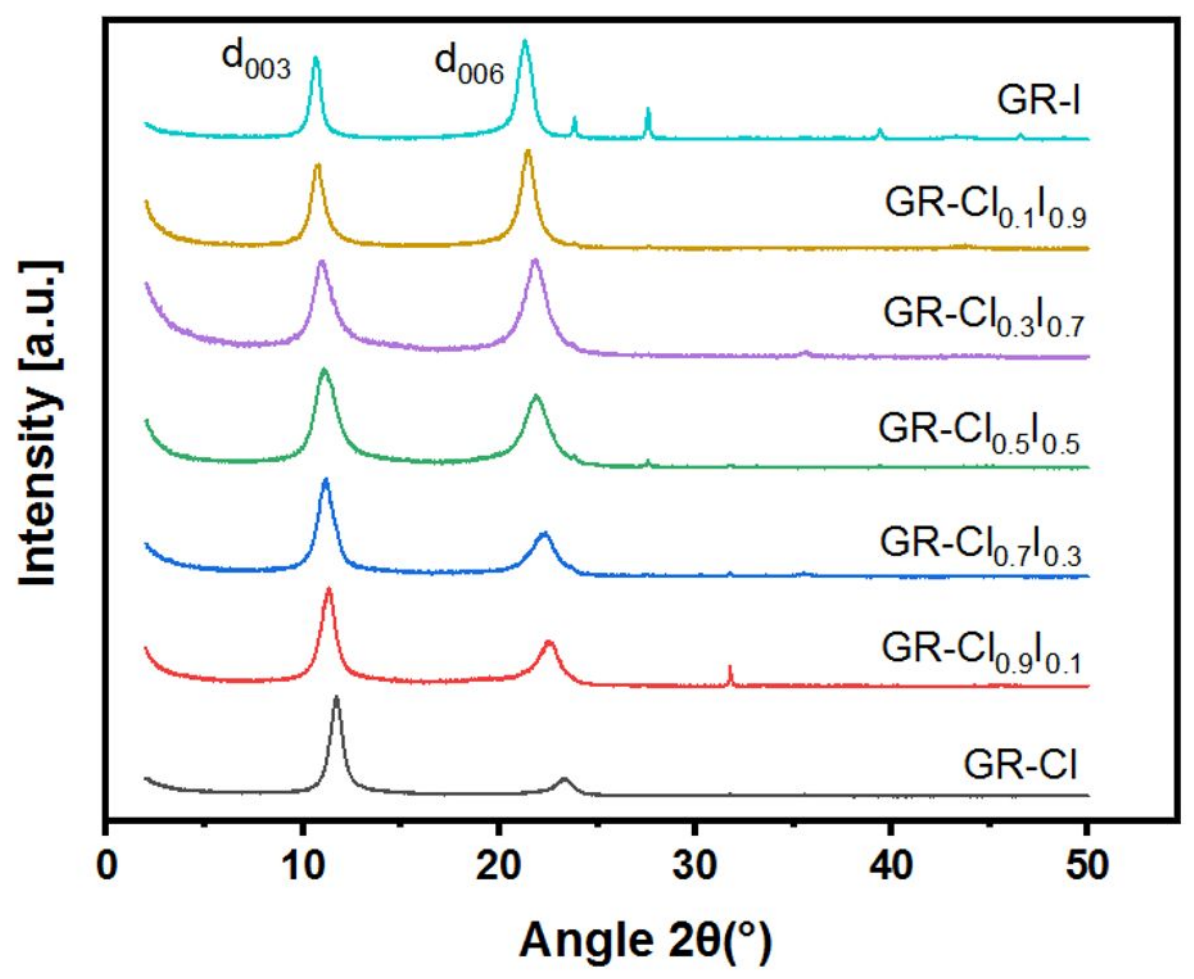

Figure S1. Experimental X-ray powder diffractogram of all GR samples. Diffractograms indicate the presence of salt formed upon drying in some samples $\left(\mathrm{NaI}\left(23.7^{\circ} / 27.5^{\circ} / 39.3^{\circ}\right)\right.$ and $\left.\mathrm{NaCl}\left(31.8^{\circ}\right)\right)$.

Fits to the powder diffractograms presented in the main manuscript were obtained considering the presence of only one green rust phase, thus suggesting the simultaneous presence of chloride and iodide in the interlayer for samples prepared from suspensions containing both halides. An alternative hypothesis would be to assume the formation of separate GR-Cl and GR-I endmembers instead of only one GR compound. To test this alternative hypothesis, (003) and 
(006) basal spacings were modeled using two reflections, one corresponding to $\mathrm{GR}-\mathrm{Cl}$ and the second to GR-I, located at positions comparable to that obtained for these pure compounds. Figure S2 shows exemplarily the result of the fit to the experimental diffractogram of GR$\mathrm{Cl}_{0.5} \mathrm{I}_{0.5}$. In the low angular range, the experimental $d_{003}$ is badly modeled using a combination of two reflections: the modeled diffractogram shows the presence of two separate reflections or one intense with a shoulder, while only one is present on the experimental diffractogram. The difference between experimental and modeled data is even more pronounced for $d_{006}$. Consequently, the formation of only separate Cl- and I-containing GR endmembers during the synthesis of the mixed GR-Cl $l_{1-\mathrm{x}} \mathrm{I}_{\mathrm{x}}$ compounds is excluded.

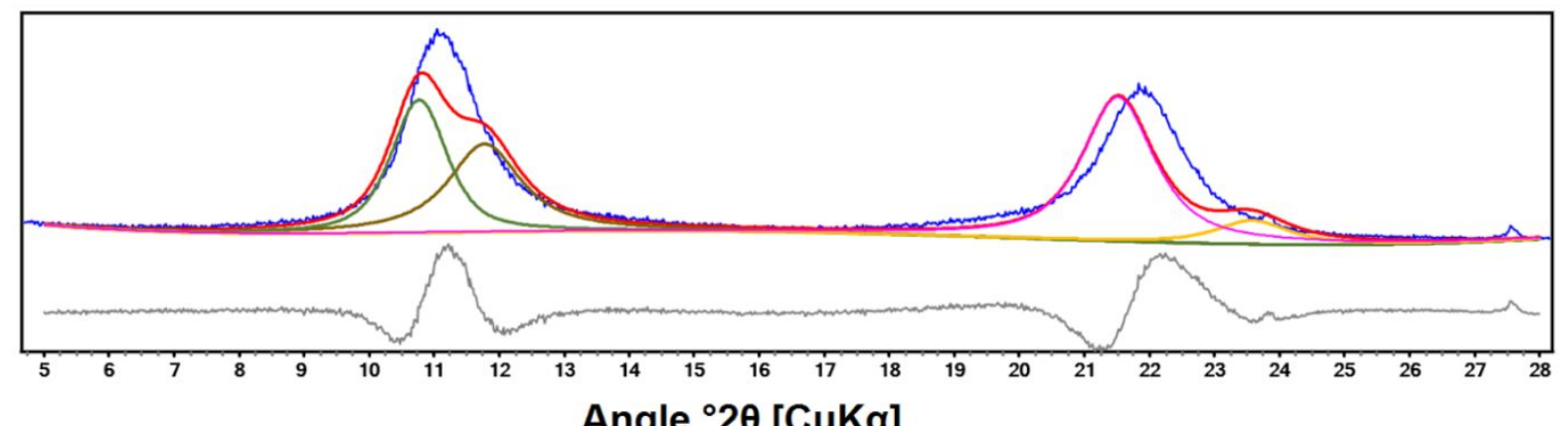
Angle ${ }^{\circ} \mathbf{2 \theta}[\mathrm{CuK \alpha}]$

Figure S2. Experimental (blue line) and modeled (red line) X-ray powder diffractogram of GR$\mathrm{Cl}_{0.5} \mathrm{I}_{0.5}$ using a mixture of GR-Cl (brown and orange line) and GR-I (green and pink line) endmembers. The grey line shows the residual fit between the experimental and modeled data. 


\section{X-ray absorption spectroscopy}

Chlorine $K$-edge $\mathrm{X}$-ray absorption spectra are rather noisy because of less beam intensity through the beryllium window, more absorption by the matrix and less emission of fluorescence radiation. Information on $\mathrm{Cl}$ speciation was provided by linear combination fitting (LCF) in the energy range $2804-2874 \mathrm{eV}$ using the Athena interface to the Ifeffit software. ${ }^{1}$ The presence of $\mathrm{FeCl}_{3}$ in the GR compounds was excluded based on the absence of intense pre-edge feature in their XANES. The considered reference compounds were crystalline sodium chloride $\left(\mathrm{NaCl}_{(\mathrm{cr})}\right)$, aqueous chloride solution $\left(\mathrm{NaCl}_{(\mathrm{aq})}\right)$, and crystalline ferrous chloride. Results of LCF are shown in Figure S3 and values are shown in Table S1.

Best fit results suggest the dominant presence of $\mathrm{FeCl}_{2}$ and $\mathrm{NaCl}_{(\mathrm{aq})}$ in $\mathrm{GR}-\mathrm{Cl}$ and in GR$\mathrm{Cl}_{0.9} \mathrm{I}_{0.1}$, and $\mathrm{FeCl}_{2}$ and $\mathrm{NaCl}_{(\mathrm{cr})}$ in all other GR compounds. However, the modeling procedure cannot be considered satisfactory because fitted data suggest the presence of a pre-edge while experimental XANES do not contain such feature and the shape of the WL is not often well captured. Overall, the XANES of the GR compounds are generally rather broad, with a width comparable to that of $\mathrm{NaCl}_{(\mathrm{aq})}$, and those being less broad contain a feature around $2838 \mathrm{eV}$, which is indicative of the presence of $\mathrm{NaCl}_{(\mathrm{cr})}$. Note that it might also be possible that another Cl-containing compound formed upon sample drying, such as $\mathrm{NaCl}_{\mathrm{x}} \mathrm{I}_{1-\mathrm{x}}$. Consequently, the absence of pre-edge feature rules out the presence of iron salt, meaning that chloride is most likely present as a hydrated anion associated with green rust and as $\mathrm{NaCl}$ and/or as $\mathrm{NaCl}_{\mathrm{x}} \mathrm{I}_{1-\mathrm{x}}$ in samples where it precipitated during sample preparation. Consequently, the speciation of chloride when associated with green rust seems comparable to that of the aqueous anions. This result is comparable to that obtained for iodide associated with GR compounds. 


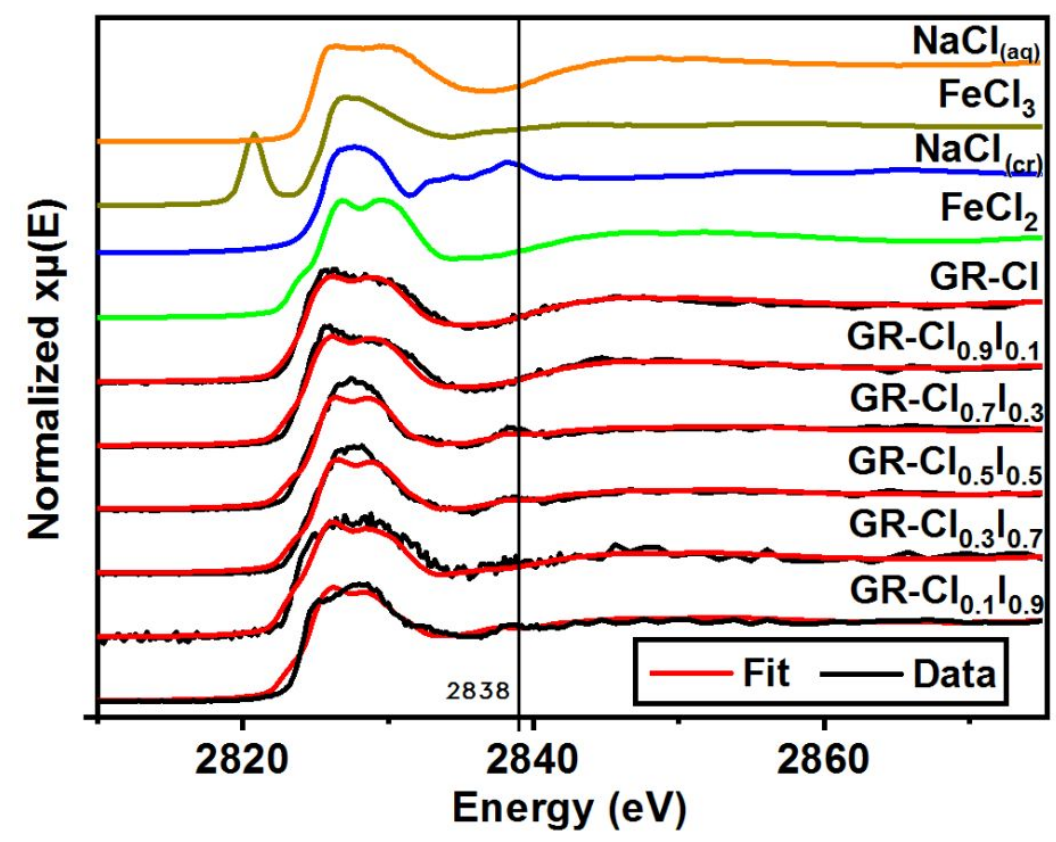

Figure S3. Experimental Cl $K$-edge XANES of the GR samples (black line) and four different reference compounds together with the linear combination fitting (red line) of GR samples. Fit results are presented in Table $\mathbf{S 1}$.

Table S1. Results of linear combination fitting to $\mathrm{Cl} K$-edge XANES of the GR samples.

\begin{tabular}{|c|c|c|c|c|}
\hline Sample & $\begin{array}{l}\text { Proportion } \\
\mathrm{NaCl}_{(\mathrm{cr})}[\%]\end{array}$ & $\begin{array}{l}\text { Proportion } \\
\mathrm{FeCl}_{2}[\%]\end{array}$ & $\begin{array}{c}\text { Proportion } \\
\mathrm{NaCl}_{(\mathbf{a q})}[\%]\end{array}$ & $\mathbf{R}_{\mathbf{f}}$ \\
\hline GR-Cl & $4(4)$ & $48(3)$ & $48.1(2.8)$ & 0.007 \\
\hline GR-Cl $\mathbf{l}_{0.9} \mathbf{I}_{0.1}$ & $6(5)$ & $60(3)$ & $34.3(3.5)$ & 0.010 \\
\hline GR-Cl $\mathbf{l}_{0.7} \mathbf{I}_{0.3}$ & $38(3)$ & $62(3)$ & - & 0.014 \\
\hline GR-Cl $_{0.5} \mathbf{I}_{0.5}$ & $31(2)$ & $69(2)$ & - & 0.011 \\
\hline GR-Cl $\mathbf{I}_{\mathbf{0 . 3}} \mathbf{I}_{\mathbf{0 . 7}}$ & $20(3)$ & $81(3)$ & - & 0.022 \\
\hline GR-Cl $\mathbf{l}_{0.1} \mathbf{I}_{0.9}$ & $35(2)$ & $65(2)$ & - & 0.013 \\
\hline
\end{tabular}

The number in parentheses indicates the uncertainty. 


\section{References}

1. Ravel, B. \& Newville, M. ATHENA, ARTEMIS , HEPHAESTUS : data analysis for X-ray absorption spectroscopy using IFEFFIT. J. Synchrotron Radiat. 12, 537-541 (2005). 